\title{
Business Intelligence in Government Driven Environment
}

\author{
Izabela Wowczko \\ Institute of Technology \\ Blanchardstown, Ireland
}

\begin{abstract}
In the aftermath of digital revolution the existence of any organisation relies upon its ability to accurately and timely answer questions. With accelerating technological advances, the HOW has been replaced with the WHAT and the WHY. Whereas many business entities struggle to survive in the word of abundance ruled by the fickle needs of their customers, governments have to stay abreast of changes to better serve the public. Despite its complexity, public sector can greatly benefit from the adoption of business intelligence that facilitates indepth analysis and informed decision making. Online services superseding traditional forms of interaction with citizens produce massive amounts of data that are sources of potentially useful information. This paper summarises the main advantages of adopting business intelligence in government driven environment. In fact, it makes valuable points for any organisation enforcing operational transparency and working for the common good.
\end{abstract}

\section{Introduction}

Although origins of the term BUSINESS INTELLIGENCE reach back over half a century [1], its recent expansion is a cross-disciplinary phenomenon. Spanning over a multitude of domains, most notably mathematics, information technology and economy - business intelligence (BI) is among top investment priorities in technology across the globe.

Industrialisation and progress in computer science have transformed many business processes. As predicted by Peter Drucker [2], human knowledge has become a fundamental component to many of them. The use of sophisticated equipment, dedicated to processing rather than manufacturing, reshaped the business landscape and positioned information as a dominant success factor. What was once considered to be merely an operational byproduct, with advanced developments in data manipulation has become the most significant asset to any organisation.

This paper aims to give a theoretical overview of business intelligence in the context of government driven environment. Section 2 covers basic definitions for better understanding of the subject. Section 3 investigates adoption of business intelligence in public sector. Section 4 concentrates on risks and constrains in adopting BI. Conclusions are summarised in section 5 .

\section{Setting the scene}

Considerable attention has been given to understanding the meaning of business intelligence and the nature of public sector. To investigate the relationship between the two, we first need to review the definitions discussed in the previous research.

\subsection{Business Intelligence}

Originated by Luhn in 1958 [1], the term BUSINESS INTELLIGENCE has over the time become a conceptual umbrella for technologies and tools fostering informed decisions in business. However, over half a decade later, it remains ambiguous and its meaning is, in a sense, circumstantial. As put forth by $\mathrm{Wu}$ [3], depending on a perspective, business intelligence can either be a process or a technology:

- In the business sense, BI is a decision making practice utilising high-quality relevant information and maximizing its business value through analysis, conclusion and assumption.

- In the information systems context, BI is a technology facilitating collecting and processing data in identifying significant correlations and patterns to support business decisions. While the common perception within the industry is that business intelligence emerged from decision support systems [3, 4], there is much controversy around its relationship to data analytics.

Great effort has been devoted to the study and development of both disciplines, and a common tendency is to blend them on conceptual and technical levels. Vendors couple BI functions traditionally understood as retrospective fact-based reporting with capabilities enabling forward-looking predictive analytics. Similarly, studies intertwine the two methods, emphasizing the importance of the relationship between past, present and future in strategic decision making [5]. Although business intelligence and data analytics are two terms used interchangeably, clear distinctions have been 
previously suggested. By way of example, Thomas H. Davenport believes BI to be a much broader concept, with analytics being 'a subset of BI based on statistics, prediction and optimization' [6]. In keeping with that differentiation, in this paper we adopt the following definition:

Business intelligence (BI) unites data, technology, analytics, and human knowledge to optimize business decisions and ultimately drive an enterprise's success. BI programs usually combine an enterprise data warehouse and a BI platform or tool set to transform data into usable, actionable business information. (The Data Warehouse Institute)

\subsection{Government Driven Environment}

Government driven environment, also referred to as public sector, is the part of economy directed by government and aimed on achieving certain social and environmental objectives. Broadly speaking, it consists of public service bodies and state-sponsored enterprises operating in commercial and noncommercial fields [7].

The main objective of the public sector is to provide governmental services to citizens on time and budget. Although methods of operation considerably vary by country, government officials are accountable for maximizing national wealth and increasing well-being of a nation. Meeting expectations of the public is a mission carried out through continuous decision making and, whether more or less critical, outcomes of that process are expected to deliver high quality results.

Previous research has demonstrated that, although effective leadership, in general, requires a specific set of capabilities, governance in public sector is heavily influenced by political pressures, and social norms and traditions [8]. One important implication of that setting is that those factors extensively constrain decision making by generating severely conditioned choices. In the result, decisions are not being optimised. Instead, they are likely to be bargained and compromised to satisfy conflicting interests [9].

In such alignment, there is a requirement for competent leaders with strong insights into organisational capabilities. One step towards leveraging the managerial effectiveness is by utilising technology to support decision-making. Successful adoption of business intelligence in the private sector has already been examined in-depth and referenced by many authors. Their findings are of direct practical relevance, and suggest a great potential for successful implementation in other areas such as public services and non-profit organisations.

\section{Adoption of BI in Public Sector}

Despite positive feedback from businesses, the public sector is still struggling to recognize the worth of business intelligence. Operating in a noncompetitive environment, it has no apparent reason for its application. Nevertheless, many transactions and events occur similarly to those in the private sector, creating analogous challenges to decisionmakers.

While research to date has focused on two primary evaluators of the performance in a public sector, namely decreasing operational costs and increasing the quality of services, there are other potential benefits to implementing advanced decision-support technology in government driven environment.

\subsection{Decision-Making Transparency}

The main purpose of business intelligence is to provide tools and methodologies for making informed decisions. After decades of reasoning based on personal experience and own judgement, decision-making has become a scientific process based in rationale and logic. Formal methods of evaluation ensure in-depth examination of alternative choices against proposed criteria.

Significance of the public sector being the pillar of social welfare and economic growth, results in accountability and specific requirements for its transparency. Compliance and openness generate public trust and confidence in administrative authorities. Whereas every organisation faces some degree of ambiguity in its operational and managerial processes, government driven environment must operate to the highest of standards. Therefore, the potential of BI in facilitating traceability of decision making cannot be overestimated.

\subsection{Policy-Making and Innovation}

Whereas a government is the principal regulatory body, public service entities serve as execution points where the strategies are delivered and executed [10]. Effectiveness of the national governance heavily relies upon relevant and forceful policies concerning individuals and organisations. In addition to a long-term planning, authorities also have legislative powers to take actions in safeguarding citizens. Capitalising on a potential of BI embodies, above all, response to current needs. Furthermore, methodologies such as real time analysis, offer protection from certain risks and social or environmental threats that cannot be timely identified by an individual decision maker. 


\subsection{Quality Assurance}

Public accountability requires formal regulations for maintaining compliance in the world of constant political change. Promotion of quality in public settings has been constantly evolving and has taken two major directions - quality of public service and professional conduct among officials. Careful monitoring of policies and their execution supports transparent, non-discriminatory and appealable procedures in national governance [10]. In the same way, comprehensive information about employment, especially with regard to senior management, identifies potential conflicts of interest that might be in breach of legislative or administrative guidelines.

In a very broad sense, public sector is a big enterprise with its management (officials), employees (civil servants), clients (citizens) and products (government services). Multitude of interactions in this heterogeneous but, even so, highly bureaucratic environment requires formal regulations. Data generated and collected from disparate sources can be utilised to get an insight into operational processes. This comprehensive view is inevitable in developing standardisation and promotion of best practices in the public sphere.

\subsection{Services Improvement}

Broad range of activities within a government and its subsidiaries produces massive sets of data. Skilful analysis cantered around selected objectives is vital in maximising the value of collected information. Providing services that are constantly in demand - such as free healthcare, social security benefits or education - have to be balanced with those reflecting specific needs of communities and businesses. Recognising hidden patterns and underling relationships can be conclusively translated onto tailored programs that will address many pressing issues. Over-servicing and underservicing are the results of faulty decisions rooted in poor judgment of reality. However, rich layers of information produced by public services allow best concepts to be developed and best outcomes to be produced [11].

\subsection{Resources Optimization}

Public sector policies and innovations directly affect economic prosperity and well-being of nations. With this intention, governments make decisions on the distribution of resources between different sectors of economy. With effective business intelligence tools such as predictive analytics in place, they are better positioned to capture opportunities and asset risks, especially in the times of economic downturn.
It cannot be ignored that many public sector activities are undertaken by private sector and nonprofit entities albeit with significant public funding. Therefore, justified allocation of resources is of uttermost importance in maintaining publicprivate sectors synergy and balancing markets in stimulating economic growth.

\subsection{Financial Performance}

Central to slow adoption of BI is the fact that the public sphere is not being profit driven. Although generating revenue through commercial services (i.e. Electricity Supply Board or An Post), the future of the public sector entities is not determined by its profitable performance. Nevertheless, the financial aspect of the government governance is of vital importance.

Pre-defined budget and cost saving operations are two primary evaluators of the financial performance among public sector entities [12]. With this in mind, there is a constant need for assessing processes and corresponding policies, and measuring their value for money in delivering services. Although in any country health and education are prerogatives in public expenditure, allocating financial resources towards high performing programs and those preferred by the citizens should be the key in funding policy.

\subsection{Workforce Management}

Governmental agencies must make careful use of their human resources, especially during economic crisis. Whereas difficult times significantly increase demand for public services in areas such as social security or education, public sector employment is expected to remain fairly steady. Maximising the value of talent in an organisation is the top objective in employment management. To address this challenge, labour data can be captured and utilised for monitoring the workforce effectiveness. Automated solutions, for example time and attendance or scheduling, leverage measurement accuracy and allow better evaluation of performance and productivity [13]. Any decision within human resources must be fairly justified as public employment is ruled not only by labour laws and local policies, but also by many union mandates. Employers must provide solid evidence that they operate in compliance, therefore increasing fairness of the evaluating criterions can by greatly supported with BI

\section{Risks and Constrains}

Several practical questions arise when dealing with business intelligence in government driven environment. Typically, any implementation of a 
new technology is subject to unavoidable risks which can affect feasibility, schedule and deliveries of the project.

\subsection{Human Engagement}

The studies of BI deployment in the private sector have shown that the relationship between IT professionals and end users is the most important determinant of a success. Because of that, understanding of the objectives and their metrics can bridge the gap between functions provided by technology and expectations of the executives. Since effective communication and genuine crossdepartmental commitment are required in building useful BI tools, it is therefore necessary for such initiatives to solicit support from decisionmakers, regardless their technical skills. Furthermore, geographical and functional decentralisation of government driven environment can be a conundrum affecting development and customisation of BI products.

Whereas employing highly skilled teams and business intelligence competency centres (BICC) is becoming a common practice in the business sector, more research is required on adopting that approach in the public sector.

\subsection{Financial Pressures}

Depending on requirements, major investments usually have to be made in order to implement and exploit full capabilities of a new technology. The sensitivity of financial matters, results from indirect measurements of performance in the public sector as explained in Section 3.6. While return on investment (ROI) can be easily calculated in evaluating financial gain in the private sector, profitability of investments in the public sector is of slightly different nature. To overcome that difficulty, sustainable return on investment (SROI) can be considered [14].

\subsection{Culture}

It is a universal trend in developed countries to strengthen the link between governments and public. In the information age, communication between policy-makers and citizens is the supreme indicator of a democratic progress. Political and cultural studies have proven that differences in cognition and world view are directly reflected in the functioning of a government apparatus [15]. Despite worldwide technological advance, misjudgements of the social, cultural or political spheres might have a major impact on the success of adopting BI. Culture determines the conditions under which private and public sectors entities operate. It also establishes limits of freedom and privacy of an individual. Therefore, cultural awareness is paramount to success of any business organisation, even more so, to the survival of a government.

\subsection{Legislature}

Through its decisions, the government is continually present in social and economic lives of its nation. The public sector itself, however, is subjected to a variety of mandates. Apart from national laws, industry rules apply to different sectors of economy. Furthermore, countries need to obey international regulations and agreements.

Whilst those arrangements are a consequence of functioning in a global economy, they also provide opportunities for growth. In fact, many recent technological developments were initiated and conducted at the international level. For example, European Union stimulates the development of common standards across many entities, including public agencies. eEurope 2005 action plan specifically establishes a framework for quality of information and speed of response in interactions between public bodies and citizens [16]. Furthermore, IT infrastructure considerably evolved with the introduction of e-government and online services creating data-rich environments that can be utilised using intelligent tools.

\subsection{Data Management and Privacy}

Creation, collection, management, use and disposal of data produced via online public service requires legislative regulations, especially with regard to privacy protection. Only commonwealth data that is authentic, accurate and reliable can be used to support accountability and decision making in the public sector. Responsible management is necessary to make the best use of data asset, whilst ensuring that the privacy of an individual remains protected [17]. The importance of maintaining public trust in handling personal information needs to be fully recognised. Certain types of data are more sensitive than others, and therefore should only be accessed and exchanged by specific job roles.

\section{Conclusions}

Generally speaking, there are very few research sources available on the use of BI in the public sector. The issue has been insufficiently investigated and further studies are required. There has been little literature published comprehending the complexity of the subject. However, many researchers examined single aspects of business intelligence or public sector, and their findings were of great support in this research.

The major drawback of the previous studies is the approach based on forcing similarities between private and public sectors. Nevertheless, most of the 
authors failed to recognize the commercial activities directed by governments such as state-owned enterprises operating in a moderately competitive environment. Therefore, the review of previous literature suggests that more focus is required on examining distinctive characteristics of the public sector and developing success measures adequate to its complex structure and unique objectives.

\section{References}

[1] Luhn, H. P., (1958). 'A business intelligence system', IBM Journal, October 1958, pp. 314 - 319.

[2] Drucker, P., (1996), Landmarks of Tomorrow: A Report on the New "Post-Modern" Word, Transaction Publishers, New Jersey.

[3] Wu, J., (2000). 'What is business intelligence?', Information Management; www.informationmanagement. com (Access date: 12 October 2014).

[4] Arnott, D., Pervan, G., (2005). 'A critical analysis of decision support systems research', Journal of Information Technology, 20, 2, June, 2005, pp. 67-87

[5] Sauter, V. L., (2010). Decision support systems for business intelligence, 2nd edn, Wiley \& Sons, New Jersey

[6] Henschen, D., (2010). 'Analytics at Work: Q\&A with Tom Davenport', InformationWeek, 04 January; www.informationweek.com (Access date: 19 October 2014)

[7] MacCarthaigh, M., (2007). The corporate governance of regional and local public service bodies in Ireland, Institute of Public Administration, Dublin

[8] Leslie, K., Canwell, A., (2010). 'Leadership at all levels: leading public sector organisations in an age of austerity', European Management Journal, 28, pp. 297 305

[9] Boselli, R., Cesarini, M., Mezzanzanica, M., (2011). 'Public service intelligence: evaluating how the public sector can exploit decision support systems', 2011 RESER Conference Papers, 7- 10 September 2011.

[10] The Organisation for Economic Co-operation and Development, (2008). Towards better measurement of government, OECD Publications, Paris

[11] Djellal, F., Gallouj, F., (2012). 'Beyond productivity measurement and strategies: performance evaluation and strategies in services', Sciences de l'Homme et de la Societe, halshs-00748099, version 1 - 4 Nov 2012; http://halshs.archives-ouvertes.fr (Access date: 13 October 2014).

[12] Boselli, R., Cesarini, M., Mezzanzanica, M., (2011). 'Framework guidelines to measure impact of business intelligence and decision support methodologies in the public sector', The Proceedings of the 11th European Conference on eGovernment, pp. 107 - 115.
[13] Lombardi, M., (2012). 'Enhancing productivity through transparency and accountability: workforce management in the public sector', Aberdeen Group; www.aberdeen.com (Access date: 15 October 2014).

[14] Colfield, A., Ellison, H., Jansen, F., McKessar, M., O'Rourke, S., Seybert, E., Williams, J., (2010). 'Sustainable return on investment. A path towards the diffusion of sustainability measurement and communication', Columbia University;www.hdrinc.com/ sites/all/files/content/brochures/files/4120-sroi-columbiabrochure.pdf (Access date: 15 October 2014).

[15] Park, J., Fables, W., Parker, K. R., Nitse P. S., (2010). 'The Role of Culture in Business Intelligence', USA International Journal of Business Intelligence Research, 1(3), 1-14, July-September 2010, DOI: 10.4018/jbir.2010070102.

[16] Sanchez, A. M., Bermejo, L. R., (2007). 'Public sector performance and efficiency in Europe: the role of public R\&D', Institute of Social and Economic Analysis, Working Papers, 01/2007; http://dspace.uah.es/dspace/bitstream/ handle/10017/6520/ public_maroto_IAESDT_2007.pdf?sequence $=1$ (Access date: 16 October 2014).

[17] Australian Government Information Management Office 2013, The Australian public service big data strategy, Commonwealth of Australia;www.finance.gov. $\mathrm{au} /$ sites/default/files/Big\%20Data\%20Strategy.pdf. (Access date: 20 October 2014). 